\title{
Numerical study on the bearing capacity of masonry platform with different number of layers
}

\author{
Ryota Hashimoto $^{\text {i) }}$, Tomofumi Koyama ${ }^{\text {ii) }}$, Mamoru Kikumoto ${ }^{\text {iii) }}$ and Mamoru Mimura ${ }^{\text {iv) }}$ \\ i) Doctoral student, Dept. of Urban Management, Kyoto Univ., Kyoto-Daigaku Katsura, Nishikyo-ku, Kyoto 615-8540, Japan. \\ ii) Associate professor, Fac. of Societal Safety Science, Kansai Univ., Hakubai-cho 7-1, Takatsuki 569-1098, Japan. \\ iii) Associate professor, Inst. of Urban Innovation, Yokohama Natl. Univ., Tokiwadai 79-5, Hodogaya-ku, Yokohama 240-8501, Japan. \\ iv) Professor, Dept. of Urban Management, Kyoto Univ., Kyoto-Daigaku Katsura, Nishikyo-ku, Kyoto 615-8540, Japan.
}

\begin{abstract}
There are many historic masonry structures collapsed due to deformation and/or failure of their foundation ground, and then, it is demanded to investigate the deterioration mechanisms and the rational restoration methods based on the geotechnical engineering. In case of the Angkor ruin in Cambodia, buildings were constructed on the foundations consist of the man-made soil embankment and the masonry stones, so-called 'platform'. Therefore, to achieve the restoration ensuring structural stability, the bearing capacity characteristics of the masonry platform in various structural conditions should be investigated. In this paper, the bearing capacity analyses of the masonry foundation with different number of layers were carried out using NMM-DDA (coupled Numerical Manifold Method and Discontinuous Deformation Analysis) enhanced with the node-based uniform strain element, and the influence of the structural features on the ultimate load was discussed.
\end{abstract}

Keywords: Bearing capacity, Masonry structure, Angkor ruin, NMM-DDA

\section{INTRODUCTION}

The Angkor ruin, a World Cultural Heritage in Cambodia, includes many masonry structures in danger of collapse due to geotechnical problems, such as uneven settlement of the foundation ground. In order to select suitable restoration methods, stability evaluation considering the mechanical interaction between the masonry building (discontinuum) and the foundation ground (continuum) is required. Especially, the bearing capacity characteristics of the foundation which consists of artificial soil embankment and masonry stones (Fig. 1), so-called 'platform' should be reflected in the design.

In case of the foundation shown in Fig. 1, the weight of the upper structure will be transmitted to both the masonry stones and the man-made embankment. Since the mechanical behaviors of such composite structures are governed by the material nonlinearity of soil and the discontinuity of the masonry stones, it is difficult to solve the failure mechanism of the platform by theoretical method.

Meanwhile, as a numerical approach, Hashimoto et al. (2017) developed a method for deformation analysis of the composite structure of the soil and the masonry stones based on NMM-DDA (coupled Numerical Manifold Method and Discontinuous Deformation Analysis). The NMM-DDA is one of the discontinuum based numerical methods, and can simulate the

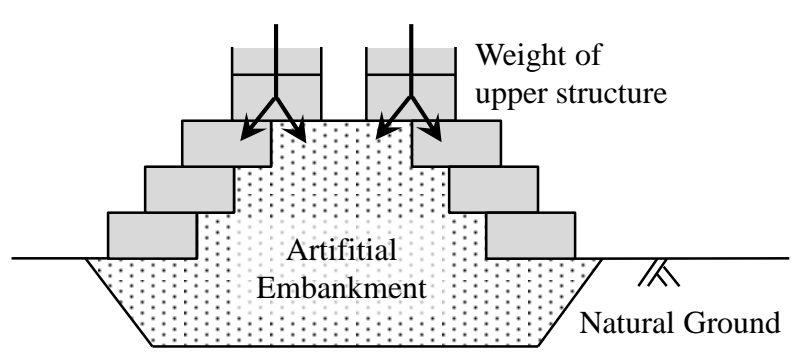

Fig. 1 Foundation of the Angkor monuments

dynamic/static behaviors of elastic materials considering mutual contact and separation at arbitrary points. Considering this advantage, Hashimoto et al. extended the NMM-DDA with the elasto-plastic constitutive models to incorporate material nonlinearity of soils, and additionally introduced the node-based uniform strain element (Dohrmann et al., 2000) to avoid the volumetric locking (Hughes, 1987) of the element. The proposed method was applied to a bearing capacity problem of a masonry platform, and the simulation results suggested that both the shear resistance of the soil and the friction strength of the masonry stones govern the ultimate bearing capacity of the platform.

However, in order to establish the quantitatively valid design method for the masonry platform, difference of the mechanical behaviors due to various structural features, such as number of layers, height of 
stones and overlapped width between the stacked stones, should be investigated. In this paper, therefore, the bearing capacity analyses of the masonry platform with different number of layers were carried out using the elasto-plastic NMM-DDA, and the influence of the structural features on the ultimate load was discussed.

\section{GOVERNING EQUATIONS AND WEAK FORM}

This section provides the governing equations considered in the elasto-plastic NMM-DDA. Since this study treats the mutual contact problem among deformable continua (see Fig.2), a system that includes $n$ discontinuous continua is hereafter assumed. The domains occupied by each continuum are named as $\Omega_{i}$ $(i=1,2, \ldots, n)$, and the boundary surfaces of $\Omega_{i}$ are named as $\Gamma_{i}$. The mechanical behavior of each continuum is governed by equation of motion

$$
\rho_{i} \ddot{\boldsymbol{u}}_{i}-\nabla \cdot \boldsymbol{\sigma}_{i}-\boldsymbol{b}_{i}=0 \quad \text { in } \Omega_{i}
$$

where subscript $i$ indicates a physical quantity of $\Omega_{i}, \rho_{i}$ is the density, $\boldsymbol{u}_{i}$ is the displacement vector, $\boldsymbol{\sigma}_{i}$ is the stress tensor, $\boldsymbol{b}_{i}$ is the body force vector, and $[\cdot]$ above a variable means material time derivative. Next, the contact between two continua $\Omega_{j}$ and $\Omega_{k}(j \neq k)$ along the boundary $\Gamma_{j k}$ is assumed, as shown in Fig. 2. In this situation, the relative normal distance $d_{n}$ between two objects must be zero on $\Gamma_{j k}$, and the tractions acting against each other on $\Gamma_{j k}$, called the contact force, must balance. Therefore, the solution has to satisfy

$$
\begin{gathered}
d_{n}=0 \quad \text { on } \Gamma_{j k}, \text { and } \\
\boldsymbol{t}_{j}+\boldsymbol{t}_{k}=0 \quad \text { on } \Gamma_{j k}
\end{gathered}
$$

in addition to Eq. (1). Furthermore, when there is friction at the contact surface, and the tangential traction force is less than the frictional strength criterion (Coulomb's criterion), the constraint condition of the tangential (shear) displacement ds between $\Omega_{j}$ and $\Omega_{k}$,

$$
d_{s}=0 \quad \text { on } \Gamma_{j k}
$$

must be satisfied. Based on the above equations and the method of weighted residual, weak form of governing equations will be derived as follows.

$$
\begin{aligned}
& \int_{\Omega_{i}} \delta \boldsymbol{u}_{i} \cdot\left(\rho_{i} \ddot{\boldsymbol{i}}_{i|++\Delta|}\right) d \Omega+\int_{\Omega_{i}} \delta \boldsymbol{\varepsilon}_{i}:\left(\boldsymbol{D}_{i}: \Delta \varepsilon_{i}\right) d \Omega \\
& -\left(\int_{\Omega_{i}} \delta \boldsymbol{u}_{i} \cdot \boldsymbol{b}_{i \mid++\Lambda} d \Omega+\int_{\Gamma_{i}} \delta \boldsymbol{u}_{i} \cdot \boldsymbol{t}_{i \mid+\Lambda} d \Gamma-\int_{\Omega_{i}} \delta \varepsilon_{i}: \boldsymbol{\sigma}_{i \mid} d \Omega\right) \\
& +\int_{\Gamma, k} \delta d_{n} \cdot p_{n} d_{n} d \Gamma+\int_{\Gamma, k} \delta d_{s} \cdot p_{s} d_{s} d \Gamma=0,
\end{aligned}
$$

where $l=j$ or $k$ and $m=j$ or $k$, and $l \neq m$; and $\Gamma_{i u}$ is displacement boundary, $\Gamma_{j t}$ is the stress boundary, $\delta \boldsymbol{u}_{i}$ is the trial function (or virtual displacement) that satisfies $\delta \boldsymbol{u}_{i}=0$ on $\Gamma_{i u}$., $\delta \boldsymbol{\varepsilon}_{i}$ is the virtual strain, $\boldsymbol{b}_{i \mid t+\Delta t}$ and $\boldsymbol{t}_{i \mid t+\Delta t}$

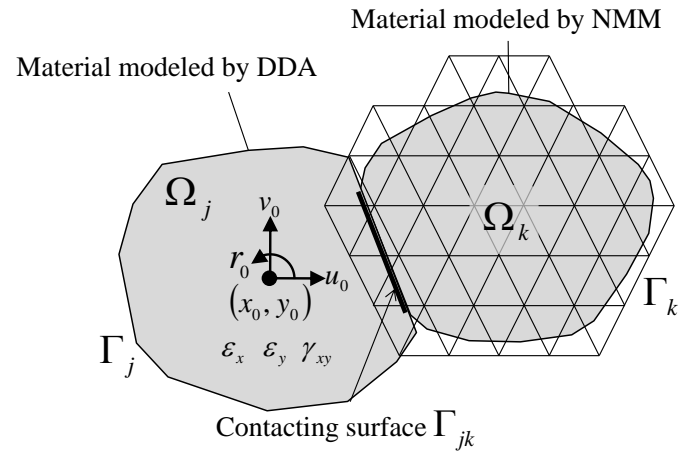

Fig. 2 Contact problem of continua

are the known body force and traction force at time $t+\Delta t$, respectively. In Eq.(5), contact conditions are satisfied using penalty method, and $p_{n}$ and $p_{s}$ are the penalty coefficient for the normal and tangential direction to contact surface. Additionally, in order to introduce elasto-plastic constitutive laws, the displacement, strain and stress are divided into known term at time $t$ and incremental unknown term between $t$ and $t+\Delta t$, and incremental form of constitutive law,

$$
\Delta \boldsymbol{\sigma}_{i}=\boldsymbol{D}_{i}: \Delta \varepsilon_{i}
$$

is applied to Eq. (5). Here, $\boldsymbol{D}_{i}$ is constitutive relation tensor. In NMM-DDA, Eq. (5) is discretized in space and time domain, and solved for each time increment.

\section{OUTLINES OF NMM-DDA WITH THE NODE-BASED UNIFORM STRAIN ELEMENT}

As described in Section 1, NMM-DDA (Miki et al., 2010) is a coupled method of two discontinuum based numerical methods, Discontinuous Deformation Analysis (Shi and Goodman, 1988) and Numerical Manifold Method (Shi, 1991). In NMM-DDA, the $n$ independent continua assumed in Section 2 are divided into those continua modeled by DDA and those modeled by NMM, as shown in Fig. 2. Since the equation of motion for each material is coupled by contact term using penalty method as shown in Eq. (5), simultaneous analysis of both domain can be achieved. The most different point between DDA and NMM is the approximation scheme of displacement field in the materials that is necessary to discretize Eq. (5). In DDA, the continua are modeled as polygons, called DDA blocks, and assuming that the stress and the strain are uniform in one DDA block, the displacement field can be expressed with three displacement variables and three strain variables, as shown in Fig. 2. Since the rotation is explicitly considered as an unknown variable, the rolling of the stones can be appropriately accounted for in analyses of the collapse behavior of masonry structures. On the other hand, in NMM, displacement field in the materials are discretized to nodal displacement of the mathematical mesh, which created 
based on finite cover concept over the physical domain as shown in Fig. 2, and consequently, NMM can represent complex displacement and strain fields in the materials, and is appropriate to analyze the detailed deformation in the ground. Based on these advantages of two methods, NMM-DDA, which integrates both methods, can model the mechanical interaction behavior between the ground and the masonry structures. After discretizing Eq. (5) of each material with DDA and NMM, the equation will be transformed into the following form.

$$
[M]\{\ddot{u}\}+\left([K]+\left[K^{\text {contact }}\right)\{\Delta u\}=\{\Delta F\}\right.
$$

Here, $[M]$ is the mass matrix, $[K]$ is the stiffness matrix, $\left[K^{\text {contact }}\right]$ is the stiffness due to contact penalty term, $\{\Delta F\}$ is the external force increment vector, $\{\ddot{u}\}$ is the acceleration vector, and $\{\Delta u\}$ is the displacement increment vector. The details about discretization and matrices can be seen in the references (Shi and Goodman, 1988; Shi, 1991; Miki et al., 2010). Using Newmark's $\beta$ method, the acceleration term in Eq. (7) can be discretized in time domain and finally we get simultaneous linear equations to be solved. Since the governing equations of all materials are combined each other by the penalty terms in the simultaneous equation, NMM-DDA can achieve the strong coupling analysis of the ground and a masonry structure satisfying the governing equations for whole domain.

However, the original NMM-DDA contains a disadvantage in solution accuracy. Since the original NMM-DDA employs constant strain triangular (CST) element for the NMM domain as shown in Fig. 2, computed results can exhibit an unrealistically stiff behavior when modeling domains containing incompressible materials (Hashimoto et al., 2017). This phenomenon is also significant when the simulation of an elasto-plastic model for soils predicts constant volume shearing at a critical state, and it is rather difficult to obtain theoretically accurate solutions. Similar phenomenon have often been reported in the finite element simulation using low-order elements, which is known as volumetric locking (Hughes, 1987), and many research about countermeasures have been done. Focusing on the theoretical similarity between NMM and FEM, Hashimoto et al. (2017) applied into NMM a method based on the so-called node-based uniform strain element (NB element), which was originally proposed by Dohrmann et al. (2000) to avoid the volumetric locking of a 3-node triangular FE mesh. The NB element is the domain which consists of the partial domain of the CST elements around a node, as shown in Fig. 3. In this method, strain components, which are usually evaluated at the center of the element, are evaluated at each node by averaging the strain components of the triangle elements sharing the node as following equation.

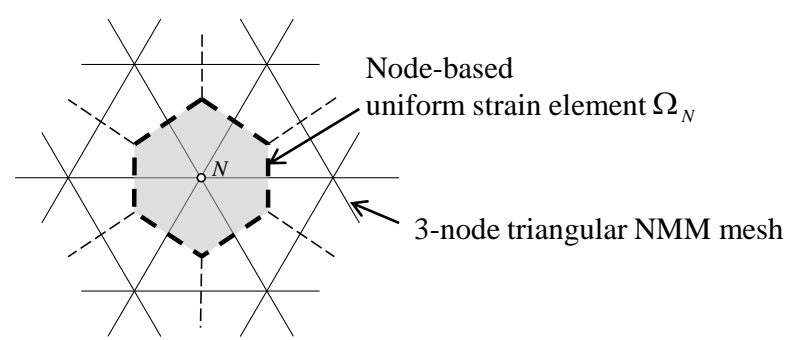

Fig. 3 Definition of the node-based uniform strain element

$$
\left\{\bar{\varepsilon}_{N}\right\}=\frac{1}{\bar{A}_{N}} \sum_{e=1}^{m_{N}} \alpha_{e}^{N} A_{e}\left\{\varepsilon_{e}\right\}
$$

Here, $\left\{\bar{\varepsilon}_{N}\right\}$ is the strain components vector at the node $N,\left\{\varepsilon_{e}\right\}$ is the strain components vector of a CST element $e, m_{N}$ is the number of CST elements containing the node $N$, and $A_{e}$ is the area of a CST element $e$, and $\bar{A}_{N}$ is the area of the NB element corresponding with the node $N . \alpha_{e}^{N}$ is the partition ratio of $A_{e}$ to the node $N$. In this study, each element is divided equally to three nodes, thus, we assume $\alpha_{e}{ }^{N}=$ $1 / 3$. The volumetric locking usually occurs when the number of constraint condition on strain due to incompressibility and/or flow rules is too large. Meanwhile, in this formulation, since the strains are integrated at the nodes, the number of integration points which corresponds with the number of constraint conditions, will be reduced by half (Dohrmann et al., 2000) from the normal CST element. Consequently, the volumetric locking can be avoided. It should be noted that the formulations of the matrices except $[K]$ in Eq. (7) and the implementation of the boundary conditions and the contact conditions are the same with the conventional NMM-DDA because this method is based on the conventional displacement field approximation in NMM.

\section{BEARING CAPACITY ANALYSIS OF MASONRY PLATFORM WITH DIFFERENT NUMBER OF LAYERS}

In the previous work by the authors (Hashimoto et al., 2017), the NMM-DDA with the node-based element was applied to a bearing capacity problem of a masonry platform, and suggested that both the shear resistance of the soil and the friction strength of the masonry stones govern the ultimate bearing capacity of the platform.

However, in order to understand failure mechanisms of the masonry platform comprehensively and establish a systematic design method, difference of the mechanical behaviors due to various structural features should be investigated. In this section, therefore, the bearing capacity analyses of the masonry platform with different number of layers were carried out using the elasto-plastic NMM-DDA, and the influence of the structural features on the ultimate load was discussed. 
Fig. 4 shows the analytical domain modeled by NMM-DDA. Here, three models with different number of layers are assumed. Each model consists of the ground and the masonry stones, and in Fig. 4(a), (b), (c), stones are stacked in four, five and six layers, respectively. Fig. 4(b) is the model employed in Hashimoto et al. (2017). The ground is modeled by NMM with the NB element, and the masonry stones are modeled by DDA. For all models, all stones are the same both in width and height, and the overlapping width between the stones is the same for all layers. The bottom boundary of the ground is fixed in all directions, and the lateral side of the ground is fixed in the horizontal direction. The values of the material properties used in the simulation are summarized in

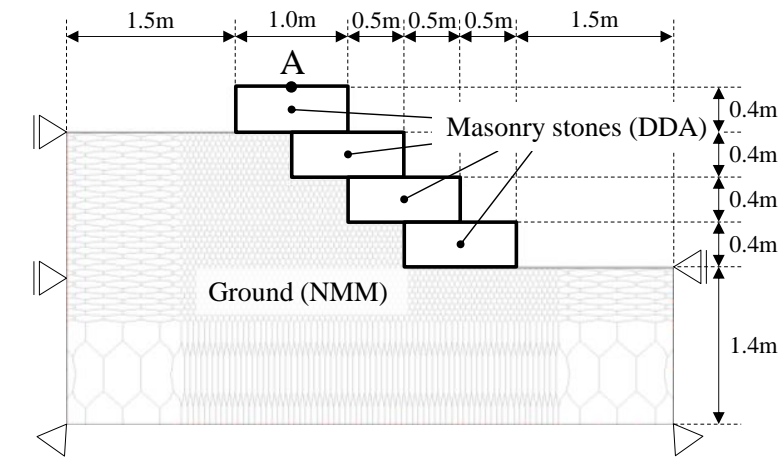

(a) Four layers

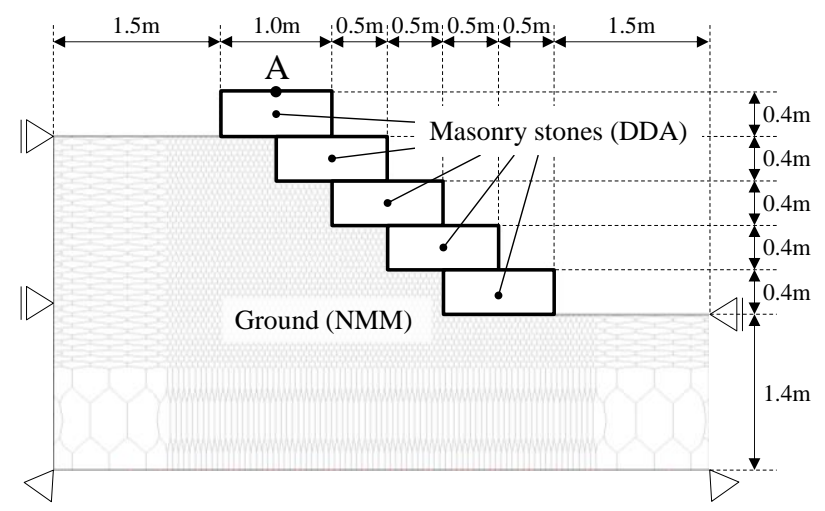

(b) Five layers

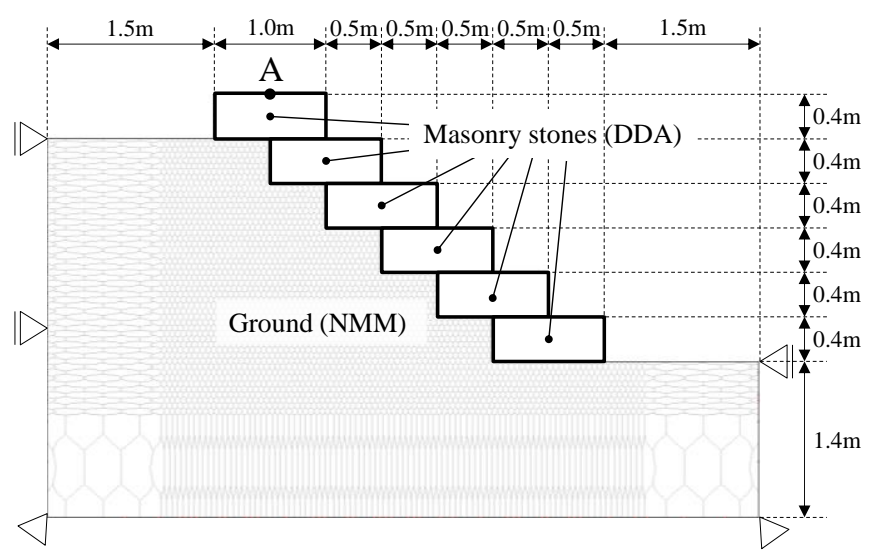

(c) Six layers

Fig. 4 Analytical domain of the masonry platform
Table 1 . The masonry stones are modeled as a linear elastic body having relatively large stiffness. The ground is modeled as an elasto-perfectly plastic material with von Mises's failure criterion, and the associated flow rule is assumed. In addition, the weight of both the masonry stones and the ground is ignored for simplicity. The parameters of the contact surface between the stones and those between the stone and the ground are assumed to be the same, as summarized in Table 2. A downward vertical displacement of $4 \mathrm{~mm}$ is applied at the center point of the upper surface of the top stone (the point A in Fig. 4) in 40,000 steps by the penalty method. Here, we define the reaction force at the loading point, which will be estimated using penalty coefficient and the residual of the applied displacement, as the bearing capacity of the masonry platform. It should be noted that the stress of the elasto-plastic material is updated using return mapping algorithm and Newton-Raphson iteration (Simo and Taylor, 1985).

Fig. 5 shows the relationship between the vertical displacement of the loading point $\mathrm{A}$ and the bearing capacity obtained from each case. In all cases, the curves showed similar behavior that the load reaches its maximum value at the vertical displacement of approximately $0.6 \mathrm{~mm}$, and the load becomes almost constant afterwards.

Table 1. Material properties used in the analyses

\begin{tabular}{lccc}
\hline & & $\begin{array}{c}\text { Ground } \\
\text { (von Mises) }\end{array}$ & $\begin{array}{c}\text { Masonry Stones } \\
\text { (Elastic) }\end{array}$ \\
\hline Young's modulus & {$[\mathrm{kPa}]$} & $1.0 \times 10^{5}$ & $1.0 \times 10^{7}$ \\
Poisson's ratio & & 0.3 & 0.2 \\
Cohesion & {$[\mathrm{kPa}]$} & 10.0 & N/A \\
\hline
\end{tabular}

Table 2. Material properties of the contact surface

\begin{tabular}{lccc}
\hline $\begin{array}{l}\text { Contact penalty } \\
\text { coefficient }\end{array}$ & Normal direction & {$[\mathrm{kN} / \mathrm{m}]$} & $1.0 \times 10^{6}$ \\
& Shear direction & {$[\mathrm{kN} / \mathrm{m}]$} & $1.0 \times 10^{3}$ \\
Friction angle & & $\left.{ }^{\circ}\right]$ & 30.0 \\
Cohesion & & {$[\mathrm{kPa}]$} & 0.0 \\
\hline
\end{tabular}

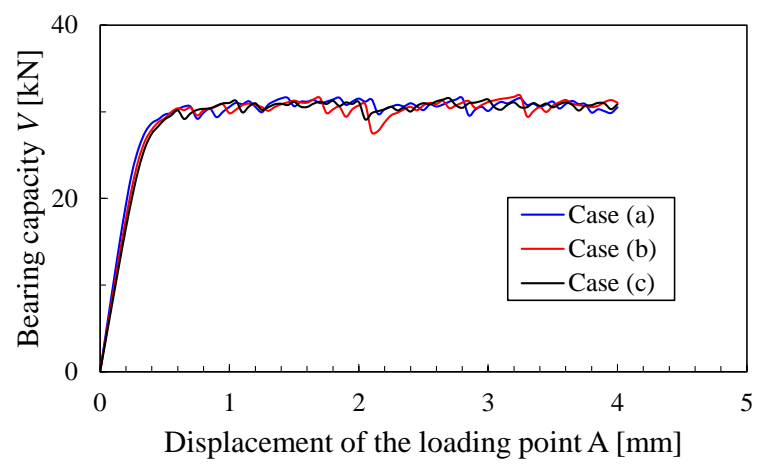

Fig. 5 Displacement-load relationship of each case 


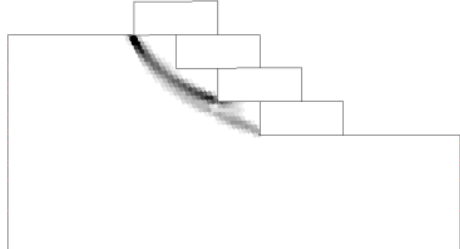

(a) Four layers

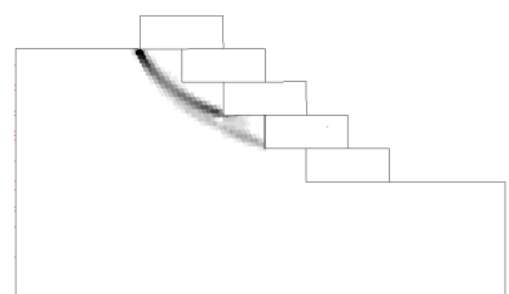

(b) Five layers

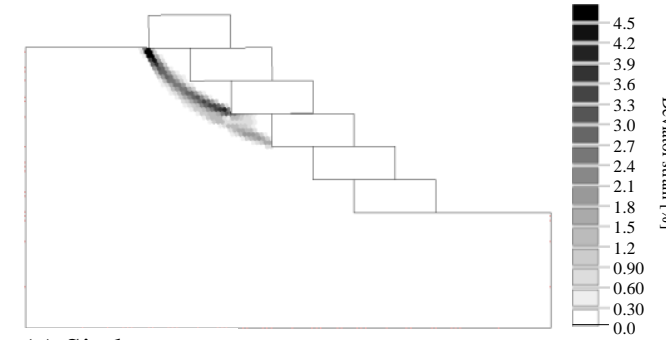

(c) Six layers

Fig. 6 Final distribution of the deviator strain

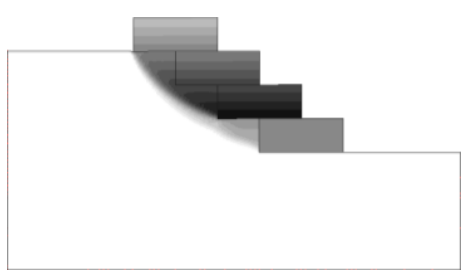

(a) Four layers

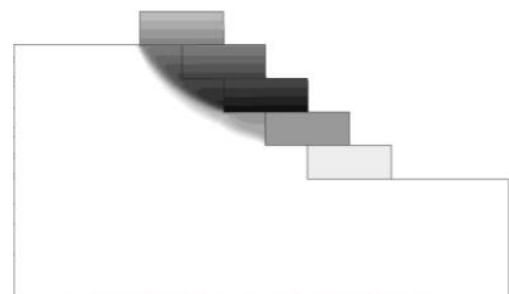

(b) Five layers

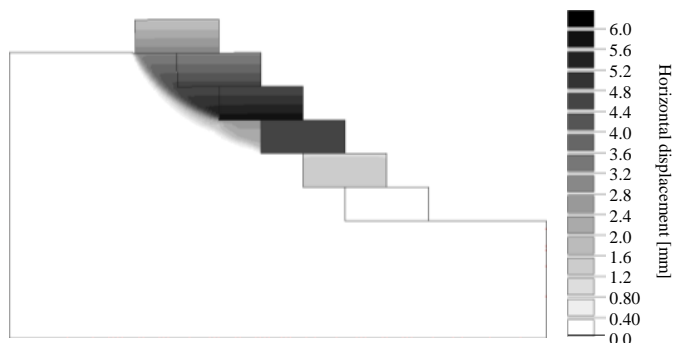

(c) Six layers

Fig. 7 Final distribution of the horizontal displacement

The final distribution of the deviator strain in the ground, and the final distribution of the horizontal displacement obtained from each case are shown in Fig. 6 and Fig. 7, respectively. From Fig. 6 two circular-shaped slip surfaces are generated from the lower left corner of the top stone, and they reach the lower left corner of the third and fourth stones from the top, respectively, in all cases. In addition, the distribution of the horizontal displacement shown in Fig. 7 indicates that the largest displacement occurred at the third stone from the top, and the displacement field becomes discontinuous between the third and fourth stones from the top, where slip failure at the boundary of the stones would occur in all cases. These results mean that both the shear resistance of the soil and the friction strength along the material sufaces governs the bearing capacity of the masonry platform structures as reported in Hashimoto et al. (2017). Additionally, based on the above results, we can conclude that the number of the layers did not affect the failure mechanism, and consequently, the bearing capacity characteristics of each case became similar.

\section{CONCLUSIONS}

In this paper, in order to investigate the influence of the structural features of the masonry platform on the bearing capacity characteristics, the bearing capacity analyses of the platform with different number of the layers were carried out using the NMM-DDA enhanced with the node-based uniform strain element. In the simulations, the failure mechanisms of the platform and the bearing capacity characteristics were almost same in all cases. Based on the results, it can be concluded that the number of the layers does not affect the bearing capacity of the masonry platform.

For the further study, the influence of other structural features, such as the overlapped width between the stones and the height of the stones should be investigated.

\section{ACKNOWLEDGEMENTS}

This work is supported by the Japan Society for the Promotion of Science (JSPS) Grant-in-Aid for JSPS Fellows [Subject No. 14J00077] provided to the first author and Grant-in-Aid for Scientific Research (B) [Subject No. 26303010] provided to the second author.

\section{REFERENCES}

1) Dohrmann, C. R., Heinstein, M. W., Jung, J., Key, S. W. and Witkowski, W. R. (2000): Node-based uniform strain elements for three-node triangular and four-node tetrahedral meshes, Int. J. Numer. Meth. Eng., 47, 1549-1568.

2) Hashimoto, R., Koyama, T., Kikumoto, M. and Mimrua, M. (2017): Method of deformation analysis for composite structures of soils and masonry stones, Computers and Geotechnics, 86, 67-84 (published online).

3) Hughes, T.J.R. (1987): The Finite Element Method: Linear Static and Dynamic Finite Element Analysis, Prentice-Hall: Englewood Cliffs, NJ.

4) Japanese Government Team for Safeguarding Angkor (JSA) (2005): Report on the conservation and restoration work of the Prasat Suor Prat Tower, OGAWAINSATSU Co., Ltd. 
5) Miki, S., Sasaki, T., Koyama, T., Nishiyama, S. and Ohnishi, Y. (2010): Development of coupled discontinuous deformation analysis and numerical manifold method (NMM-DDA), Int. J. Comput. Methods, 7(1), 1-20.

6) Shi, G.H. and Goodman, R.E. (1988): Discontinuous deformation analysis-a new method for computing stress, strain and sliding of block systems, Key Questions in Rock Mechanics, Cundall et al. eds., Balkema, 381-393.

7) Shi, G.H. (1991): Manifold method of material analysis, Transactions of the 9th Army Conference on Applied Mathematics and Computing, Report No. 92-1, U.S. Army Research Office.

8) Simo, J.C. and Taylor, R.L. (1985): Consistent tangent operators for rate-independent elastoplasticity, Comput.Methods in Appl.Mech.Eng., 48, 101-118. 